\title{
ORE'S THEOREM ON CYCLIC SUBFACTOR PLANAR ALGEBRAS AND BEYOND
}

\author{
SEBASTIEN PALCOUX
}

\begin{abstract}
Ore proved that a finite group is cyclic if and only if its subgroup lattice is distributive. Now, since every subgroup of a cyclic group is normal, we call a subfactor planar algebra cyclic if all its biprojections are normal and form a distributive lattice. The main result generalizes one side of Ore's theorem and shows that a cyclic subfactor is singly generated in the sense that there is a minimal 2-box projection generating the identity biprojection. We conjecture that this result holds without assuming the biprojections to be normal, and we show that it is true for small lattices. We finally exhibit a dual version of another theorem of Ore and a non-trivial upper bound for the minimal number of irreducible components for a faithful complex representation of a finite group.
\end{abstract}

\section{INTRODUCTION}

Vaughan Jones proved in [10] that the set of possible values for the index $|M: N|$ of a subfactor $(N \subseteq M)$ is

$$
\left\{4 \cos ^{2}\left(\frac{\pi}{n}\right) \mid n \geq 3\right\} \sqcup[4, \infty] .
$$

We observe that it is the disjoint union of a discrete series and a continuous series. Moreover, for a given intermediate subfactor $N \subseteq P \subseteq M$, $|M: N|=|M: P| \cdot|P: N|$, therefore by applying a kind of Eratosthenes sieve, we get that a subfactor of index in the discrete series or in the interval $(4,8)$, except the countable set of numbers composed of numbers in the discrete series, can't have a non-trivial intermediate subfactor. A subfactor without non-trivial intermediate subfactor is called maximal [4]. For example, any subfactor of index in $(4,3+\sqrt{5})$ is maximal; (except $A_{\infty}$ ) there are exactly 19 irreducible subfactor planar algebras for this interval (see [1,13]), the first example is the Haagerup subfactor [23]. Thanks to Galois correspondence [19], a finite group subfactor, $\left(R^{G} \subseteq R\right)$ or $(R \subseteq R \rtimes G)$, is maximal if and only if it is a prime order cyclic group subfactor (i.e. $G=\mathbb{Z} / p$ with $p$ prime).

Key words and phrases. von Neumann algebra; subfactor; planar algebra; biprojection; distributive lattice; finite group; representation. 
Thus we can say that the maximal subfactors are an extension of the prime numbers.

Question 1.1. What could be the extension of the natural numbers?

To answer this question, we need to find a natural class of subfactors, that we will call the "cyclic subfactors", satisfying:

(1) Every maximal subfactor is cyclic.

(2) A finite group subfactor is cyclic iff the group is cyclic.

An old and little known theorem published in 1938 by the Norwegian mathematician Øystein Ore states that:

Theorem $1.2([20])$. A finite group $G$ is cyclic if and only if its subgroup lattice $\mathcal{L}(G)$ is distributive.

Firstly, the intermediate subfactor lattice of a maximal subfactor is obviously distributive. Next, by Galois correspondence, the intermediate subfactor lattice of a finite group subfactor is exactly the subgroup lattice (or its reversal) of the group; but distributivity is invariant under reversal, so (1) and (2) hold by Ore's theorem. Now an abelian group, and a fortiori a cyclic group, admits only normal subgroups; but $T$. Teruya generalized in [25] the notion of normal subgroup by the notion of normal intermediate subfactor, so:

Definition 1.3. A finite index irreducible subfactor is cyclic if all its intermediate subfactors are normal and form a distributive lattice.

Note that an irreducible finite index subfactor $(N \subseteq M)$ admits a finite lattice $\mathcal{L}(N \subseteq M)$ of intermediate subfactors by [26], as for the subgroup lattice of a finite group. Moreover, a finite group subfactor remembers the group by [9]. Section 4.1 exhibits plenty of examples of cyclic subfactors: of course the cyclic group subfactors and the (irreducible finite index) maximal subfactors; moreover, up to equivalence, exactly 23279 among 34503 inclusions of groups of index $<30$, give a cyclic subfactor. The class of cyclic subfactors is stable under dual, intermediate, free composition and certain tensor products. Now the natural problem about cyclic subfactors is to understand in what sense they are "singly generated". To answer this question, we extend the following theorem of Ore.

Theorem 1.4 (O. Ore, [20]). If an interval of finite groups $[H, G]$ is distributive, then $\exists g \in G$ such that $\langle H, g\rangle=G$.

Theorem 1.5. An irreducible subfactor planar algebra whose biprojections are central and form a distributive lattice, has a minimal 2-box projection generating the identity biprojection (i.e. w-cyclic subfactor). 
But "normal" means "bicentral", so a cyclic subfactor planar algebra is w-cyclic. The converse is false, a group subfactor $\left(R^{G} \subseteq R\right)$ is cyclic if and only if $G$ is cyclic, and is w-cyclic if and only if $G$ is linearly primitive (take $G=S_{3}$ ). That's why we have chosen the name w-cyclic (i.e. weakly cyclic). We conjecture that Theorem 1.5 holds without the assumption "central".

Conjecture 1.6. An irreducible subfactor planar algebra with a distributive biprojection lattice is w-cyclic.

It is true if the lattice has less than 32 elements (and so, at index $<32$ ). Now the group-theoretic reformulation of Conjecture 1.6 for the planar algebra $\mathcal{P}\left(R^{G} \subseteq R^{H}\right)$, gives a dual version of Theorem 1.4.

Conjecture 1.7. If the interval of finite groups $[H, G]$ is distributive then $\exists V$ irreducible complex representation of $G$ such that $G_{\left(V^{H}\right)}=H$.

In general, we deduce a non-trivial upper bound for the minimal number of minimal central projections generating the identity biprojection. For $\mathcal{P}\left(R^{G} \subseteq R\right)$, this gives a non-trivial upper bound for the minimal number of irreducible components for a faithful complex representation of $G$. It is a bridge linking combinatorics and representations in the theory of finite groups. This paper is a short version of [22].

\section{Contents}

1. Introduction

2. Ore's theorem on finite groups

2.1. Basics in lattice theory

2.2. Ore's theorem on distributive intervals of finite groups

3. Subfactor planar algebras and biprojections

3.1. Basics on the 2-box space

3.2. On the biprojections

3.3. Intermediate planar algebras and 2-box spaces 7

4. Ore's theorem on subfactor planar algebras 7

4.1. The cyclic subfactor planar algebras 7

4.2. The w-cyclic subfactor planar algebras

4.3. The main result 12

5. Extension for small distributive lattices 13

6. Applications 15

6.1. A non-trivial upper bound $\quad 15$

6.2. Back to the finite groups theory $\quad 16$

7. Acknowledgments 18

References $\quad 19$ 


\section{ORE'S THEOREM ON FINITE GROUPS}

2.1. Basics in lattice theory. A lattice $(L, \wedge, \vee)$ is a poset $L$ in which every two elements $a, b$ have a unique supremum (or join) $a \vee b$ and a unique infimum (or meet) $a \wedge b$. Let $G$ be a finite group. The set of subgroups $K \subseteq G$ forms a lattice, denoted by $\mathcal{L}(G)$, ordered by $\subseteq$, with $K_{1} \vee K_{2}=\left\langle K_{1}, K_{2}\right\rangle$ and $K_{1} \wedge K_{2}=K_{1} \cap K_{2}$. A sublattice of $(L, \wedge, \vee)$ is a subset $L^{\prime} \subseteq L$ such that $\left(L^{\prime}, \wedge, \vee\right)$ is also a lattice. Consider $a, b \in L$ with $a \leq b$, then the interval $[a, b]$ is the sublattice $\{c \in L \mid a \leq c \leq b\}$. Any finite lattice admits a minimum and a maximum, denoted by $\hat{0}$ and $\hat{1}$. An atom (resp. coatom) is a minimal (resp. maximal) element in $L \backslash\{\hat{0}\}$ (resp. $L \backslash\{\hat{1}\}$ ). The top interval of a finite lattice $L$ is the interval $[t, \hat{1}]$, with $t$ the meet of all the coatoms. The height of a finite lattice $L$ is the greatest length of a (strict) chain. A lattice is distributive if the join and meet operations distribute over each other.

Remark 2.1. Distributivity is stable under taking sublattice, reversal, direct product and concatenation.

A distributive lattice is called boolean if any element $b$ admits a unique complement $b^{\complement}$ (i.e. $b \wedge b^{\complement}=\hat{0}$ and $b \vee b^{\complement}=\hat{1}$ ). The subset lattice of $\{1,2, \ldots, n\}$, with union and intersection, is called the boolean lattice $\mathcal{B}_{n}$ of rank $n$. Any finite boolean lattice is isomorphic to some $\mathcal{B}_{n}$.

Lemma 2.2. The top interval of a finite distributive lattice is boolean.

Proof. See [24, items a-i p254-255] which uses Birkhoff's representation theorem (a finite lattice is distributive iff it embeds into some $\mathcal{B}_{n}$ ).

A lattice with a boolean top interval will be called top boolean (and its reversal, bottom boolean). See [24] for more details on lattice basics.

2.2. Ore's theorem on distributive intervals of finite groups. Øystein Ore proved the following result in [20, Theorem 4, p267].

Theorem 2.3. A finite group $G$ is cyclic if and only if its subgroup lattice $\mathcal{L}(G)$ is distributive.

Proof. $(\Leftarrow)$ : It is just a particular case of Theorem 2.5 with $H=\{e\}$. $(\Rightarrow)$ : A finite cyclic group $G=\mathbb{Z} / n$ has exactly one subgroup of order $d$, denoted by $\mathbb{Z} / d$, for every divisor $d$ of $n$. Now $\mathbb{Z} / d_{1} \vee \mathbb{Z} / d_{2}=$ $\mathbb{Z} / \mathrm{lcm}\left(d_{1}, d_{2}\right)$ and $\mathbb{Z} / d_{1} \wedge \mathbb{Z} / d_{2}=\mathbb{Z} / \operatorname{gcd}\left(d_{1}, d_{2}\right)$, but lcm and gcd distribute other each over, the result follows.

Definition 2.4. An interval of finite groups $[H, G]$ is said to be $H$ cyclic if there is $g \in G$ such that $\langle H, g\rangle=G$. Note that $\langle H, g\rangle=\langle H g\rangle$. 
Ore extended one side of Theorem 2.3 to the interval of finite groups [20, Theorem 7] for which we will give our own proof (which is a grouptheoretic reformulation of the proof of Theorem 4.26):

Theorem 2.5. A distributive interval $[H, G]$ is $H$-cyclic.

Proof. The proof follows from the claims below and Lemma 2.2.

Claim: Let $M$ be a maximal subgroup of $G$. Then $[M, G]$ is $M$-cyclic. Proof: For $g \in G$ with $g \notin M$, we have $\langle M, g\rangle=G$ by maximality.

Claim: A boolean interval $[H, G]$ is $H$-cyclic.

$\underline{\text { Proof: }}$ Let $M$ be a coatom in $[H, G]$, and $M^{\complement}$ be its complement. By the previous claim and induction on the height of the lattice, we can assume $[H, M]$ and $\left[H, M^{\complement}\right]$ both to be $H$-cyclic, i.e. there are $a, b \in G$ such that $\langle H, a\rangle=M$ and $\langle H, b\rangle=M^{\complement}$. For $g=a b, a=g b^{-1}$ and $b=a^{-1} g$, so $\langle H, a, g\rangle=\langle H, g, b\rangle=\langle H, a, b\rangle=M \vee M^{\complement}=G$. Now, $\langle H, g\rangle=\langle H, g\rangle \vee H=\langle H, g\rangle \vee\left(M \wedge M^{\complement}\right)$ but by distributivity $\left.\left.\langle H, g\rangle \vee\left(M \wedge M^{\complement}\right)=(\langle H, g\rangle \vee M\rangle\right) \wedge\left(\langle H, g\rangle \vee M^{\complement}\right\rangle\right)$. So $\langle H, g\rangle=\langle H, a, g\rangle \wedge\langle H, g, b\rangle=G$. The result follows.

Claim: $[H, G]$ is $H$-cyclic if its top interval $[K, G]$ is $K$-cyclic.

Proof: Consider $g \in G$ with $\langle K, g\rangle=G$. For any coatom $M \in[H, G]$, we have $K \subseteq M$ by definition, and so $g \notin M$, then a fortiori $\langle H, g\rangle \nsubseteq$ $M$. It follows that $\langle H, g\rangle=G$.

\section{SUBFACTOR PLANAR ALGEBRAS AND BIPROJECTIONS}

For the notions of subfactor, subfactor planar algebra and basic properties, we refer to [11,12,15]. See also [22, Section 3] for a short introduction. A subfactor planar algebra is of finite index by definition.

3.1. Basics on the 2-box space. Let $(N \subseteq M)$ be a finite index irreducible subfactor. The $n$-box spaces $\mathcal{P}_{n,+}$ and $\mathcal{P}_{n,-}$ of the planar algebra $\mathcal{P}=\mathcal{P}(N \subseteq M)$, are $N^{\prime} \cap M_{n-1}$ and $M^{\prime} \cap M_{n}$. Let $R(a)$ be the range projection of $a \in \mathcal{P}_{2,+}$. We define the relations $a \preceq b$ by $R(a) \leq R(b)$, and $a \sim b$ by $R(a)=R(b)$. Let $e_{1}:=e_{N}^{M}$ and $i d:=e_{M}^{M}$ be the Jones and the identity projections in $\mathcal{P}_{2,+}$. Note that $\operatorname{tr}\left(e_{1}\right)=|M: N|^{-1}=\delta^{-2}$ and $\operatorname{tr}(i d)=1$. Let $\mathcal{F}: \mathcal{P}_{2, \pm} \rightarrow \mathcal{P}_{2, \mp}$ be the Fourier transform (90 rotation), $\bar{a}:=\mathcal{F}(\mathcal{F}(a))$ the contragredient of $a \in \mathcal{P}_{2, \pm}$, and $a * b=\mathcal{F}\left(\mathcal{F}^{-1}(a) \cdot \mathcal{F}^{-1}(b)\right)$ the coproduct of $a, b \in \mathcal{P}_{2, \pm}$.

Lemma 3.1. Let $a, b, c, d$ be positive operators of $\mathcal{P}_{2,+}$. Then

(1) $a * b$ is also positive, 
(2) $[a \preceq b$ and $c \preceq d] \Rightarrow a * c \preceq b * d$,

(3) $a \preceq b \Rightarrow\langle a\rangle \leq\langle b\rangle$,

(4) $a \sim b \Rightarrow\langle a\rangle=\langle b\rangle$.

Proof. It is precisely [18, Theorem 4.1 and Lemma 4.8] for (1) and (2). Next, if $a \preceq b$, then by (2), for any integer $k, a^{* k} \preceq b^{* k}$, and hence $\forall n$

$$
\sum_{k=1}^{n} a^{* k} \preceq \sum_{k=1}^{n} b^{* k},
$$

then $\langle a\rangle \leq\langle b\rangle$ by Definition 3.8. Finally, (4) is immediate from (3).

The next lemma follows by irreducibility (i.e. $\mathcal{P}_{1,+}=\mathbb{C}$ ).

Lemma 3.2. Let $p, q \in \mathcal{P}_{2,+}$ be projections. Then

$$
e_{1} \preceq p * \bar{q} \Leftrightarrow p q \neq 0 .
$$

Note that if $p \in \mathcal{P}_{2,+}$ is a projection then $\bar{p}$ is also a projection.

Lemma 3.3. Let $a, b, c \in \mathcal{P}_{2,+}$ be projections with $c \preceq a * b$. Then $\exists a^{\prime} \preceq c * \bar{b}$ and $\exists b^{\prime} \preceq \bar{a} * c$ such that $a^{\prime}, b^{\prime}$ are projections and $a a^{\prime}, b b^{\prime} \neq 0$.

Proof. By using Lemmas 3.1 and 3.2 , and

$$
e_{1} \preceq c * \bar{c} \preceq(a * b) * \bar{c}=a *(b * \bar{c}) .
$$

We can also apply [18, Lemma 4.10].

\subsection{On the biprojections.}

Definition 3.4 ([18, Definition 2.14). A biprojection is a projection $b \in \mathcal{P}_{2, \pm}$ with $\mathcal{F}(b)$ a multiple of a projection.

Note that $e_{1}=e_{N}^{M}$ and $i d=e_{M}^{M}$ are biprojections.

Theorem 3.5 ([4] p212). A projection $b$ is a biprojection if and only if it is the Jones projection $e_{K}^{M}$ of an intermediate subfactor $N \subseteq K \subseteq M$.

Therefore the set of biprojections is a lattice of the form $\left[e_{1}, i d\right]$.

Theorem 3.6. An operator $b$ is a biprojection if and only if

$$
e_{1} \leq b=b^{2}=b^{\star}=\bar{b}=\lambda b * b \text {, with } \lambda^{-1}=\delta \operatorname{tr}(b) .
$$

Proof. See [17, items 0-3 p191] and [18, Theorem 4.12].

Lemma 3.7. Consider $a_{1}, a_{2}, b \in \mathcal{P}_{2,+}$ with $b$ a biprojection. Then

$$
\left(b \cdot a_{1} \cdot b\right) *\left(b \cdot a_{2} \cdot b\right)=b \cdot\left(a_{1} *\left(b \cdot a_{2} \cdot b\right)\right) \cdot b=b \cdot\left(\left(b \cdot a_{1} \cdot b\right) * a_{2}\right) \cdot b,
$$

$\left(b * a_{1} * b\right) \cdot\left(b * a_{2} * b\right)=b *\left(a_{1} \cdot\left(b * a_{2} * b\right)\right) * b=b *\left(\left(b * a_{1} * b\right) \cdot a_{2}\right) * b$.

Proof. By exchange relations [17] for $b$ and $\mathcal{F}(b)$. 
Definition 3.8. Consider $a \in \mathcal{P}_{2,+}$ positive, and let $p_{n}$ be the range projection of $\sum_{k=1}^{n} a^{* k}$. By finiteness, there exists $N$ such that for all $m \geq N, p_{m}=p_{N}$, which is a biprojection [18, Lemma 4.14], denoted $\langle a\rangle$, called the biprojection generated by $a$. It is the smallest biprojection $b \succeq a$. For $S$ a finite set of positive operators, let $\langle S\rangle$ be the biprojection $\left\langle\sum_{s \in S} s\right\rangle$, it is the smallest biprojection $b$ such that $b \succeq s, \forall s \in S$.

\subsection{Intermediate planar algebras and 2-box spaces.}

Let $N \subseteq K \subseteq M$ be an intermediate subfactor. The planar algebras $\mathcal{P}(N \subseteq K)$ and $\mathcal{P}(K \subseteq M)$ can be derived from $\mathcal{P}(N \subseteq M)$, see [3, 16].

Theorem 3.9. Consider the intermediate subfactors

$$
N \subseteq P \subseteq K \subseteq Q \subseteq M
$$

Then there are two isomorphisms of von Neumann algebras

$$
\begin{gathered}
l_{K}: \mathcal{P}_{2,+}(N \subseteq K) \rightarrow e_{K}^{M} \mathcal{P}_{2,+}(N \subseteq M) e_{K}^{M}, \\
r_{K}: \mathcal{P}_{2,+}(K \subseteq M) \rightarrow e_{K}^{M} * \mathcal{P}_{2,+}(N \subseteq M) * e_{K}^{M},
\end{gathered}
$$

for usual,$+ \times$ and ()$^{\star}$, such that

$$
l_{K}\left(e_{P}^{K}\right)=e_{P}^{M} \text { and } r_{K}\left(e_{Q}^{M}\right)=e_{Q}^{M} .
$$

Moreover, the coproduct $*$ is also preserved by these maps, but up to a multiplicative constant, $|M: K|^{1 / 2}$ for $l_{K}$ and $|K: N|^{-1 / 2}$ for $r_{K}$. Then, $\forall m \in\left\{l_{K}^{ \pm 1}, r_{K}^{ \pm 1}\right\}, \forall a_{i}>0$ in the domain of $m, m\left(a_{i}\right)>0$ and

$$
\left\langle m\left(a_{1}\right), \ldots, m\left(a_{n}\right)\right\rangle=m\left(\left\langle a_{1}, \ldots, a_{n}\right\rangle\right) .
$$

Proof. Immediate from [3] or [16], using Lemma 3.7.

Notations 3.10. Let $b_{1} \leq b \leq b_{2}$ be the biprojections $e_{P}^{M} \leq e_{K}^{M} \leq e_{Q}^{M}$. We define $l_{b}:=l_{K}$ and $r_{b}:=r_{K} ;$ also $\mathcal{P}\left(b_{1}, b_{2}\right):=\mathcal{P}(P \subseteq Q)$ and

$$
\left|b_{2}: b_{1}\right|:=\operatorname{tr}\left(b_{2}\right) / \operatorname{tr}\left(b_{1}\right)=|Q: P| .
$$

\section{ORE'S THEOREM ON SUBFACTOR PLANAR ALGEBRAS}

\subsection{The cyclic subfactor planar algebras.}

In this subsection, we define the class of cyclic subfactor planar algebras, we show that it contains plenty of examples, and we prove that it is stable under dual, intermediate, free composition and certain tensor products. Let $\mathcal{P}$ be an irreducible subfactor planar algebra.

Definition 4.1 ([25]). A biprojection b is normal if it is bicentral (i.e. $b$ and $\mathcal{F}(b)$ are central).

Definition 4.2. An irreducible subfactor planar algebra is said to be

- distributive if its biprojection lattice is distributive. 
- Dedekind if all its biprojections are normal.

- cyclic if it is both Dedekind and distributive.

Moreover, we call a subfactor cyclic if its planar algebra is cyclic.

Examples 4.3. A group subfactor is cyclic if and only if the group is cyclic; every maximal subfactor is cyclic, in particular every 2supertransitive subfactor, as the Haagerup subfactor [2,66,23], is cyclic. Up to equivalence, exactly 23279 among 34503 inclusions of groups of index $<30$, give a cyclic subfactor (more than $65 \%$ ).

Definition 4.4. Let $G$ be a finite group and $H$ a subgroup. The core $H_{G}$ is the largest normal subgroup of $G$ contained in $H$. The subgroup $H$ is called core-free if $H_{G}=\{1\}$; in this case the interval $[H, G]$ is also called core-free. Two intervals of finite groups $[A, B]$ and $[C, D]$ are called equivalent if there is a group isomorphism $\phi: B / A_{B} \rightarrow D / C_{D}$ such that $\phi\left(A / A_{B}\right)=C / C_{D}$.

Remark 4.5. A finite group subfactor remembers the group [9], but a finite group-subgroup subfactor does not remember the equivalence class of the interval in general. A counterexample was found by V.S. Sunder and $V$. Kodiyalam [14], the intervals $\left[\langle(1234)\rangle, S_{4}\right]$ and $\left[\langle(12)(34)\rangle, S_{4}\right]$ are not equivalent whereas their corresponding subfactors are isomorphic; but thanks to the complete characterization [7] by M. Izumi, it remembers the interval in the maximal case, because the intersection of a core-free maximal subgroup with an abelian normal subgroup is trivial.

Theorem 4.6. The free composition of irreducible finite index subfactors has no extra intermediate.

Proof. See [18, Theorem 2.22].

Corollary 4.7. The class of finite index irreducible cyclic subfactors is stable under free composition.

Proof. By Theorem 4.6, the intermediate subfactor lattice of a free composition is the concatenation of the lattice of the two components (and see Remark 2.1). By Theorem 3.9 and Lemma 3.7, the biprojections remain normal.

The following theorem was proved in the 2-supertransitive case by Y. Watatani [26, Proposition 5.1]. The general case was conjectured by the author, but specified and proved after a discussion with $\mathrm{F}$. Xu.

Theorem 4.8. Let $\left(N_{i} \subset M_{i}\right), i=1,2$, be irreducible finite index subfactors. Then

$$
\mathcal{L}\left(N_{1} \subset M_{1}\right) \times \mathcal{L}\left(N_{2} \subset M_{2}\right) \subsetneq \mathcal{L}\left(N_{1} \otimes N_{2} \subset M_{1} \otimes M_{2}\right)
$$


if and only if there are intermediate subfactors $N_{i} \subseteq P_{i} \subset Q_{i} \subseteq M_{i}$, $i=1,2$, such that $\left(P_{i} \subset Q_{i}\right)$ is depth 2 and isomorphic to $\left(R^{\mathbb{A}_{i}} \subset R\right)$, with $\mathbb{A}_{2} \simeq \mathbb{A}_{1}^{\text {cop }}$ the (very simple) Kac algebra $\mathbb{A}_{1}$ with the opposite coproduct.

Proof. Consider the intermediate subfactors

$$
N_{1} \otimes N_{2} \subseteq P_{1} \otimes P_{2} \subset R \subset Q_{1} \otimes Q_{2} \subseteq M_{1} \otimes M_{2}
$$

with $R$ not of tensor product form, $P_{1} \otimes P_{2}$ and $Q_{1} \otimes Q_{2}$ the closest (below and above resp.) to $R$ among those of tensor product form. Now using [27, Proposition 3.5 (2)], $\left(P_{i} \subseteq Q_{i}\right), i=1,2$, are depth 2 , their corresponding Kac algebras, $\mathbb{A}_{i}, i=1,2$, are very simple and $\mathbb{A}_{2} \simeq \mathbb{A}_{1}^{c o p}$ [27, Definition 3.6 and Proposition 3.10]. The converse is given by [27, Theorem 3.14].

Remark 4.9. By Theorem 4.8 and Remark [2.1, the class of (finite index irreducible) cyclic subfactors is stable under certain tensor products (i.e. if there is no cop-isomorphic depth 2 intermediate), and by Theorem 3.9 and Lemma 3.7, the biprojections remain normal.

Lemma 4.10. If a subfactor is cyclic then the intermediate and dual subfactors are also cyclic.

Proof. By Remark 2.1, Theorem 3.9 and Lemma 3.7.

A subfactor as $(R \subseteq R \rtimes G)$ or $\left(R^{G} \subseteq R\right.$ ) is called a "group subfactor". Then, the following lemma justifies the choice of the word "cyclic".

Lemma 4.11. A cyclic "group subfactor" is a "cyclic group" subfactor.

Proof. By Galois correspondence, if a "group subfactor" is cyclic then the subgroup lattice is distributive, and so the group is cyclic by Ore's Theorem 2.3. The normal biprojections of a group subfactor corresponds to the normal subgroups [25], but every subgroup of a cyclic group is normal.

Problem 4.12. Is a depth 2 irreducible finite index cyclic subfactor, a cyclic group subfactor?

The answer could be no because the following fusion ring (discovered by the author [21]), the first known to be simple integral and nontrivial, could be the Grothendieck ring of a "maximal" Kac algebra of dimension 210 and type $(1,5,5,5,6,7,7)$.

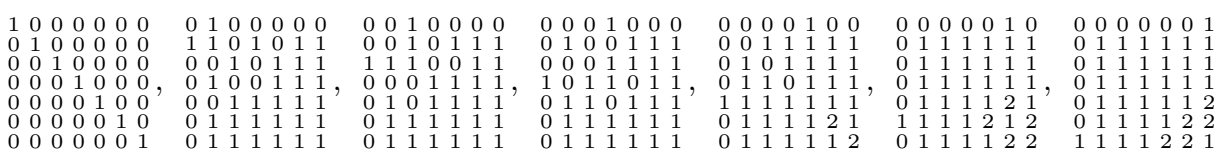




\subsection{The w-cyclic subfactor planar algebras.}

Let $\mathcal{P}$ be an irreducible subfactor planar algebra.

Theorem 4.13. Let $p \in \mathcal{P}_{2,+}$ be a minimal central projection. Then there exists $v \leq p$ minimal projection such that $\langle v\rangle=\langle p\rangle$.

Proof. If $p$ is a minimal projection, then it is ok. Else, let $b_{1}, \ldots, b_{n}$ be the coatoms of $\left[e_{1},\langle p\rangle\right]$ ( $n$ is finite by [26]). If $p \npreceq \sum_{i=1}^{n} b_{i}$, then $\exists u \leq p$ minimal projection such that $u \not \leq b_{i} \forall i$, so that $\langle u\rangle=\langle p\rangle$. Else $p \preceq \sum_{i=1}^{n} b_{i}$ (with $n>1$, otherwise $p \leq b_{1}$ and $\langle p\rangle \leq b_{1}$, contradiction). Let $E_{i}=$ range $\left(b_{i}\right)$ and $F=$ range $(p)$, then $F=\sum_{i} E_{i} \cap F$ (because $p$ is a minimal central projection) with $1<n<\infty$ and $E_{i} \cap F \subsetneq F \forall i$ (otherwise $\exists i$ with $p \leq b_{i}$, contradiction), so $\operatorname{dim}\left(E_{i} \cap F\right)<\operatorname{dim}(F)$ and there exists $U \subseteq F$ one-dimensional subspace such that $U \nsubseteq E_{i} \cap F \forall i$, and so a fortiori $U \nsubseteq E_{i} \forall i$. It follows that $u=p_{U} \leq p$ is a minimal projection such that $\langle u\rangle=\langle p\rangle$.

Thanks to Theorem 4.13, we can give the following definition:

Definition 4.14. The planar algebra $\mathcal{P}$ is weakly cyclic (or w-cyclic) if it satisfies one of the following equivalent assertions:

- $\exists u \in \mathcal{P}_{2,+}$ minimal projection such that $\langle u\rangle=i d$.

- $\exists p \in \mathcal{P}_{2,+}$ minimal central projection such that $\langle p\rangle=i d$.

We call a subfactor $w$-cyclic if its planar algebra is w-cyclic.

The following remark justifies the choice of the word "w-cyclic".

Remark 4.15. By Corollary 6.12, a finite group subfactor $\left(R^{G} \subset R\right)$ is w-cyclic if and only if $G$ is linearly primitive, which is strictly weaker than cyclic (see for example $S_{3}$ ), nevertheless the notion of $w$-cyclic is a singly generated notion in the sense that "there is a minimal projection generating the identity biprojection". We can also see the weakness of this assumption by the fact that the minimal projection does not necessarily generate a basis for the set of positive operators, but just the support of it, i.e. the identity.

Question 4.16. Is a cyclic subfactor planar algebra w-cyclic?

The answer is yes by Theorem 4.27.

Let $\mathcal{P}=\mathcal{P}(N \subseteq M)$ be an irreducible subfactor planar algebra. Take an intermediate subfactor $N \subseteq K \subseteq M$ and its biprojection $b=e_{K}^{M}$.

Lemma 4.17. Let $\mathcal{A}$ be a $\star$-subalgebra of $\mathcal{P}_{2,+}$. Then any element $x \in \mathcal{A}$ is positive in $\mathcal{A}$ if and only if it is positive in $\mathcal{P}_{2,+}$.

Proof. If $x$ is positive in $\mathcal{A}$, then it is of the form $a a^{\star}$, with $a \in \mathcal{A}$, but $a \in \mathcal{P}_{2,+}$ also, so $x$ is positive in $\mathcal{P}_{2,+}$. Conversely, if $x$ is positive in 
$\mathcal{P}_{2,+}$ then $\langle x y \mid y\rangle=\operatorname{tr}\left(y^{\star} x y\right) \geq 0$, for any $y \in \mathcal{P}_{2,+}$, so in particular, for any $y \in \mathcal{A}$, which means that $x$ is positive in $\mathcal{A}$.

Note that Lemma 4.17 will be applied to $\mathcal{A}=b \mathcal{P}_{2,+} b$ or $b * \mathcal{P}_{2,+} * b$.

Proposition 4.18. The planar algebra $\mathcal{P}\left(e_{1}, b\right)$ is $w$-cyclic if and only if there is a minimal projection $u \in \mathcal{P}_{2,+}$ such that $\langle u\rangle=b$.

Proof. The planar algebra $\mathcal{P}(N \subseteq K)$ is w-cyclic if and only if there is a minimal projection $x \in \mathcal{P}_{2,+}(N \subseteq K)$ such that $\langle x\rangle=e_{K}^{K}$, if and only if $l_{K}(\langle x\rangle)=l_{K}\left(e_{K}^{K}\right)$, if and only if $\langle u\rangle=e_{K}^{M}$ (by Theorem 3.9), with $u=l_{K}(x)$ a minimal projection in $e_{K}^{M} \mathcal{P}_{2,+} e_{K}^{M}$ and in $\mathcal{P}_{2,+}$.

Lemma 4.19. For any minimal projection $x \in \mathcal{P}_{2,+}(b, i d), r_{b}(x)$ is positive and for any minimal projection $v \preceq r_{b}(x)$, there is $\lambda>0$ such that $b * v * b=\lambda r_{b}(x)$.

Proof. Firstly, $x$ is positive, so by Theorem [3.9, $r_{b}(x)$ is also positive. For any minimal projection $v \preceq r_{b}(x)$, we have $b * v * b \preceq r_{b}(x)$, because

$$
b * v * b \preceq b * r_{b}(x) * b=b * b * u * b * b \sim b * u * b=r_{b}(x),
$$

by Lemma 3.1(2) and with $u \in \mathcal{P}_{2,+}$. Now by Lemma 3.1(1), $b * v * b>0$, so $r_{b}^{-1}(b * v * b)>0$ also, and by Theorem 3.9 ,

$$
r_{b}^{-1}(b * v * b) \preceq x .
$$

But $x$ is a minimal projection, so by positivity, $\exists \lambda>0$ such that

$$
r_{b}^{-1}(b * v * b)=\lambda x .
$$

It follows that $b * v * b=\lambda r_{b}(x)$.

Lemma 4.20. Consider $v \in \mathcal{P}_{2,+}$ positive. Then $\langle b * v * b\rangle=\langle b, v\rangle$.

Proof. Firstly, by Definition [3.8, $b * v * b \preceq\langle b, v\rangle$, so by Lemma 3.1](3), $\langle b * v * b\rangle \leq\langle b, v\rangle$. Next $e_{1} \leq b$ and $x * e_{1}=e_{1} * x=\delta^{-1} x$, so

$$
v=\delta^{2} e_{1} * v * e_{1} \preceq b * v * b .
$$

Moreover by Theorem [3.6, $\bar{v} \preceq\langle b * v * b\rangle$, but by Lemma 3.2,

$$
\bar{v} * b * v * b \succeq \bar{v} * e_{1} * v * b \sim \bar{v} * v * b \succeq e_{1} * b \sim b .
$$

Then $b, v \leq\langle b * v * b\rangle$, so we also have $\langle b, v\rangle \leq\langle b * v * b\rangle$.

Proposition 4.21. The planar algebra $\mathcal{P}(b, i d)$ is $w$-cyclic if and only if there is a minimal projection $v \in \mathcal{P}_{2,+}$ such that $\langle b, v\rangle=i d$ and $r_{b}^{-1}(b * v * b)$ is a positive multiple of a minimal projection.

Proof. The planar algebra $\mathcal{P}(K \subseteq M)$ is w-cyclic if and only if there is a minimal projection $x \in \mathcal{P}_{2,+}(K \subseteq M)$ such that $\langle x\rangle=e_{M}^{M}$, if and only if $r_{K}(\langle x\rangle)=r_{K}\left(e_{M}^{M}\right)$, if and only if $\left\langle r_{K}(x)\right\rangle=e_{M}^{M}$ by Theorem 3.9. The results follows by Lemmas 4.19 and 4.20 . 


\subsection{The main result.}

Let $\mathcal{P}$ be an irreducible subfactor planar algebra.

Lemma 4.22. A maximal subfactor planar algebra is w-cyclic.

Proof. By maximality $\langle u\rangle=i d$ for any minimal projection $u \neq e_{1}$.

Definition 4.23. The top intermediate subfactor planar algebra is the intermediate associated to the top interval of the biprojection lattice.

Lemma 4.24. An irreducible subfactor planar algebra is w-cyclic if its top intermediate is so.

Proof. Let $b_{1}, \ldots, b_{n}$ be the coatoms in $\left[e_{1}, i d\right]$ and $t=\bigwedge_{i=1}^{n} b_{i}$. By assumption and Proposition 4.21, there is a minimal projection $v \in \mathcal{P}_{2,+}$ with $\langle t, v\rangle=i d$. If $\exists i$ such that $v \leq b_{i}$, then $\langle t, v\rangle \leq b_{i}$, contradiction. So $\forall i, v \not \leq b_{i}$ and then $\langle v\rangle=i d$.

Definition 4.25. Let $h(\mathcal{P})$ be the height of the biprojection lattice $\left[e_{1}, i d\right]$. Note that $h(\mathcal{P})<\infty$ because the index is finite.

Theorem 4.26. If the biprojections in $\mathcal{P}_{2,+}$ are central and form a distributive lattice, then $\mathcal{P}$ is w-cyclic.

Proof. By Lemma 4.10, we can make an induction on $h(\mathcal{P})$. If $h(\mathcal{P})=$ 1 , then we apply Lemma 4.22, Now suppose that the theorem holds for $h(\mathcal{P})<n$, we will prove it for $h(\mathcal{P})=n \geq 2$. By Lemmas 2.2 and 4.24, we can assume the biprojection lattice to be boolean. For $b$ in the open interval $\left(e_{1}, i d\right)$, its complementary $b^{\complement}$ (see Section 2.1) is also in $\left(e_{1}, i d\right)$. By induction and Proposition 4.18, there are minimal projections $u, v$ such that $b=\langle u\rangle$ and $b^{\complement}=\langle v\rangle$. Take any minimal projection $c \preceq u * v$, then

$$
\langle c\rangle=\langle c\rangle \vee e_{1}=\langle c\rangle \vee\left(b \wedge b^{\complement}\right)=\langle c\rangle \vee(\langle u\rangle \wedge\langle v\rangle),
$$

so by distributivity

$$
\langle c\rangle=(\langle c\rangle \vee\langle u\rangle) \wedge(\langle c\rangle \vee\langle v\rangle)=\langle c, u\rangle \wedge\langle c, v\rangle .
$$

Then by Lemma 3.3, $\langle c\rangle=\left\langle u^{\prime}, c, v\right\rangle \wedge\left\langle u, c, v^{\prime}\right\rangle$ with $u^{\prime}, v^{\prime}$ minimal projections and $u u^{\prime}, v v^{\prime} \neq 0$, so in particular the central support $Z\left(u^{\prime}\right)=Z(u)$ and $Z\left(v^{\prime}\right)=Z(v)$. Now by assumption, every biprojection is central, so $u \leq Z\left(u^{\prime}\right) \leq\left\langle u^{\prime}, c, v\right\rangle$ and $v \leq Z\left(v^{\prime}\right) \leq\left\langle u, c, v^{\prime}\right\rangle$, then $\langle c\rangle=i d$.

Theorem 4.27. A cyclic subfactor planar algebra is w-cyclic.

Proof. Immediate by Theorem 4.26 because a normal biprojection is by definition bicentral, so a fortiori central. 


\section{EXTENSION FOR SMALL DISTRIBUTIVE LATTICES}

We extend Theorem 4.26 without assuming the biprojections to be central, but for distributive lattices with less than 32 elements. Because the top lattice of a distributive lattice is boolean (Lemma 2.2), we can reduce the proof to $\mathcal{B}_{n}$ with $n<5$.

Definition 5.1. An irreducible subfactor planar algebra is said to be boolean (or $\mathcal{B}_{n}$ ) if its biprojection lattice is boolean (of rank $n$ ).

Proposition 5.2. An irreducible subfactor planar algebra such that the coatoms $b_{1}, \ldots, b_{n} \in\left[e_{1}, i d\right]$ satisfy $\sum_{i} \frac{1}{\left|i d: b_{i}\right|} \leq 1$, is $w$-cyclic.

Proof. Firstly, by Lemmas 4.22 and 4.24, we can assume that $n>1$. By Definition $\left|i d: b_{i}\right|=\frac{\operatorname{tr}(i d)}{\operatorname{tr}\left(b_{i}\right)}$ so by assumption $\sum_{i} \operatorname{tr}\left(b_{i}\right) \leq \operatorname{tr}(i d)$. If $\sum_{i} b_{i} \sim i d$ then $\sum_{i} b_{i} \geq i d$, but $\sum_{i} \operatorname{tr}\left(b_{i}\right) \leq \operatorname{tr}(i d)$ so $\sum_{i} b_{i}=i d$. Now $\forall i e_{1} \leq b_{i}$, so $n e_{1} \leq \sum_{i} b_{i}=i d$, contradiction with $n>1$. So $\sum_{i} b_{i} \prec i d$, which implies the existence of a minimal projection $u \not \leq b_{i} \forall i$, which means that $\langle u\rangle=i d$.

Remark 5.3. The converse is false, $(R \subset R \rtimes \mathbb{Z} / 30)$ is a counterexample, because $1 / 2+1 / 3+1 / 5=31 / 30>1$.

Corollary 5.4. An irreducible subfactor planar algebra with at most two coatoms in $\left[e_{1}, i d\right]$ is w-cyclic.

Proof. $\sum_{i} \frac{1}{\left|i d: b_{i}\right|} \leq 1 / 2+1 / 2$, the result follows by Proposition 5.2 .

Examples 5.5. Every $\mathcal{B}_{2}$ subfactor planar algebra is w-cyclic.

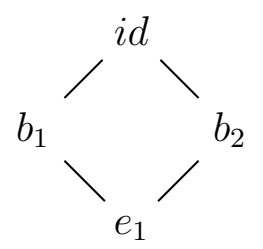

Lemma 5.6. Let $u, v \in \mathcal{P}_{2,+}$ be minimal projections. If $v \not \leq\langle u\rangle$ then $\exists c \preceq u * v$ and $\exists w \preceq \bar{u} * c$ minimal projections such that $w \not \leq\langle u\rangle$.

Proof. Assume that $\forall c \preceq u * v$ and $\forall w \preceq \bar{u} * c$ we have $w \leq\langle u\rangle$. Now there are minimal projections $\left(c_{i}\right)_{i}$ and $\left(w_{i, j}\right)_{i, j}$ such that $u * v \sim \sum_{i} c_{i}$ and $\bar{u} * c_{i} \sim \sum_{j} w_{i, j}$. It follows that $u * v \sim \sum_{i, j} w_{i, j} \preceq\langle u\rangle$, but

$$
v \sim e_{1} * v \preceq(\bar{u} * u) * v=\bar{u} *(u * v) \preceq\langle u\rangle,
$$

which is in contradiction with $v \not \leq\langle u\rangle$.

For the distributive case, we can upgrade Proposition 5.2 as follows: 
Theorem 5.7. A distributive subfactor planar algebra such that the coatoms $b_{1}, \ldots, b_{n} \in\left[e_{1}, i d\right]$ satisfy $\sum_{i} \frac{1}{\left|i d: b_{i}\right|} \leq 2$, is w-cyclic.

Proof. By Lemmas 2.2 and 4.24, we can assume the subfactor planar algebra to be boolean.

If $K:=\bigwedge_{i, j, i \neq j}\left(b_{i} \wedge b_{j}\right)^{\perp} \neq 0$, then consider $u \leq K$ a minimal projection, and $Z(u)$ its central support. If $\langle Z(u)\rangle=i d$, then we are ok. Else $\exists i$ such that $\langle u\rangle=\langle Z(u)\rangle=b_{i}$. But $b_{i}^{\complement}$ is an atom in $\left[e_{1}, i d\right]$, so there is a minimal projection $v$ such that $b_{i}^{\complement}=\langle v\rangle$. Recall that $b_{i} \wedge b_{i}^{\complement}=e_{1}$, so $v \not \leq\langle u\rangle$, and by Lemma [5.6, there are minimal projections $c \preceq u * v$ and $w \preceq \bar{u} * c$ such that $w \npreceq\langle u\rangle$ (and $\langle u, w\rangle=i d$ by maximality). By Lemma 3.3, $\exists u^{\prime} \preceq c * \bar{u}$ with $Z\left(u^{\prime}\right)=Z(u)$ and $u^{\prime} \not \perp u$, but $u \leq K$ so $\forall j \neq i, u^{\prime} \not \leq b_{i} \wedge b_{j}$, now $u^{\prime} \leq Z(u) \leq b_{i}$, so $\left\langle u^{\prime}\right\rangle=b_{i}$. Using distributivity (as for Theorem 4.26) we conclude by

$$
\langle c\rangle=\langle u, c\rangle \wedge\langle c, v\rangle \geq\langle u, w\rangle \wedge\left\langle u^{\prime}, v\right\rangle=i d \wedge i d=i d .
$$

Else $K=0$, but $\forall i,\left(b_{i} \wedge b_{j}\right)^{\perp} \geq b_{j}^{\perp}$, so $\forall i, \bigwedge_{j \neq i} b_{j}^{\perp}=0$. Let $p_{1}, \ldots, p_{r}$ be the minimal central projections. Then $b_{i}=\bigoplus_{s=1}^{r} p_{i, s}$ with $p_{i, s} \leq p_{s}$ and $p_{i, 1}=p_{1}=e_{1}$. Now $b_{i}^{\perp}=\bigoplus_{s=1}^{r}\left(p_{s}-p_{i, s}\right)$, so by assumption,

$$
0=\bigwedge_{j \neq i} \bigoplus_{s=1}^{r}\left(p_{s}-p_{j, s}\right)=\bigoplus_{s=1}^{r} \bigwedge_{j \neq i}\left(p_{s}-p_{j, s}\right), \forall i .
$$

It follows that for all $i$ and $s, p_{s}=\bigvee_{j \neq i} p_{j, s}$, so $\operatorname{tr}\left(p_{s}\right) \leq \sum_{j \neq i} \operatorname{tr}\left(p_{j, s}\right)$. Now if $\exists s$ such that $\forall i p_{i, s}<p_{s}$, then $\left\langle p_{s}\right\rangle=i d$, which is ok; else $\forall s, \exists i$ with $p_{i, s}=p_{s}$, but $\sum_{j \neq i} \operatorname{tr}\left(p_{j, s}\right) \geq \operatorname{tr}\left(p_{s}\right)$, so $\sum_{j} \operatorname{tr}\left(p_{j, s}\right) \geq 2 \operatorname{tr}\left(p_{s}\right)$. Then

$$
\sum_{i} \operatorname{tr}\left(b_{i}\right) \geq n \cdot \operatorname{tr}\left(e_{1}\right)+2 \sum_{s \neq 1} \operatorname{tr}\left(p_{s}\right)=2 \operatorname{tr}(i d)+(n-2) \operatorname{tr}\left(e_{1}\right) .
$$

Now $\left|i d: b_{i}\right|=\operatorname{tr}(i d) / \operatorname{tr}\left(b_{i}\right)$, so

$$
\sum_{i} \frac{1}{\left|i d: b_{i}\right|} \geq 2+\frac{n-2}{\left|i d: e_{1}\right|}
$$

which contradicts the assumption, because we can assume $n>2$ by Corollary 5.4. The result follows.

Remark 5.8. The converse is false because there exists w-cyclic distributive subfactor planar algebras with $\sum_{i} \frac{1}{\left|i d: b_{i}\right|}>2$. For example, the subfactor $\left(R \rtimes S_{2}^{n} \subset R \rtimes S_{3}^{n}\right)$ is w-cyclic and $\mathcal{B}_{n}$, but $\sum_{i} \frac{1}{\left|i d: b_{i}\right|}=n / 3$.

Corollary 5.9. Every $\mathcal{B}_{n}$ subfactor planar algebra with $|i d: b| \geq n / 2$, for any coatom $b \in\left[e_{1}, i d\right]$, is w-cyclic. Then $\forall n \leq 4$, any $\mathcal{B}_{n}$ subfactor planar algebra is w-cyclic. 
Proof. By assumption (following the notations of Theorem 5.7])

$$
\sum_{i} \frac{1}{\left|i d: b_{i}\right|} \leq \sum_{i} \frac{2}{n}=2 .
$$

But $|i d: b| \geq 2$, so any $n \leq 4$ works.

Corollary 5.10. A distributive subfactor planar algebra having less than 32 biprojections (or of index $<32$ ), is w-cyclic.

Proof. In this case, the top of $\left[e_{1}, i d\right]$ is boolean of rank $n<5$, because $32=2^{5}$; the result follows by Lemma 4.24 and Corollary 5.9 .

Conjecture 5.11. A distributive subfactor planar algebra is w-cyclic.

By Lemmas 2.2 and 4.24, we can reduce Conjecture 5.11 to the boolean case, and then extend it to the top boolean case.

Remark 5.12. The converse of Conjecture 5.11 is false, because the group $S_{3}$ is linearly primitive but not cyclic (see Corollary 6.12).

Problem 5.13. What is the natural additional assumption (A) such that $\mathcal{P}$ is distributive if and only if it is w-cyclic and satisfies (A)?

Assuming Conjecture 5.11 and using Remark 2.1, we get:

Conjecture 5.14. For any distributive subfactor planar algebra $\mathcal{P}$ and any biprojection $b \in \mathcal{P}_{2,+}$, the planar algebras $\mathcal{P}\left(e_{1}, b\right), \mathcal{P}(b, i d)$ and their duals are $w$-cyclic.

Remark 5.15. The converse is false because the interval $\left[S_{2}, S_{4}\right]$, proposed by Zhengwei Liu, gives a counter-example.

Remark 5.16. A cyclic subfactor planar algebra satisfies Conjecture 5.14 (thanks to Theorem 4.27 and Lemma 4.10).

Problem 5.17. Is a Dedekind subfactor planar algebra $\mathcal{P}$ distributive if and only if for any biprojection $b \in \mathcal{P}_{2,+}$, the planar algebras $\mathcal{P}\left(e_{1}, b\right)$, $\mathcal{P}(b, i d)$ and their duals are $w$-cyclic?

\section{Applications}

6.1. A non-trivial upper bound. For any irreducible subfactor planar algebra $\mathcal{P}$, we exhibit a non-trivial upper bound for the minimal number of minimal 2-box projections generating the identity biprojection. We will use the notations of Section 3.3.

Lemma 6.1. Let $b^{\prime}<b$ be biprojections. If $\mathcal{P}\left(b^{\prime}, b\right)$ is $w$-cyclic, then there is a minimal projection $u \in \mathcal{P}_{2,+}$ such that $\left\langle b^{\prime}, u\right\rangle=b$. 
Proof. Consider the isomorphisms of von Neumann algebras

$$
l_{b}: \mathcal{P}_{2,+}\left(e_{1}, b\right) \rightarrow b \mathcal{P}_{2,+} b
$$

and, with $a=l_{b}^{-1}\left(b^{\prime}\right)$,

$$
r_{a}: \mathcal{P}_{2,+}\left(b^{\prime}, b\right) \rightarrow a * \mathcal{P}_{2,+}\left(e_{1}, b\right) * a .
$$

Then, by assumption, the planar algebra $\mathcal{P}\left(b^{\prime}, b\right)$ is w-cyclic, so by Proposition 4.21, $\exists u^{\prime} \in \mathcal{P}_{2,+}\left(e_{1}, b\right)$ minimal projection such that

$$
\left\langle a, u^{\prime}\right\rangle=l_{b}^{-1}(b) .
$$

Then by applying the map $l_{b}$ and Theorem 3.9 , we get

$$
b=\left\langle l_{b}(a), l_{b}\left(u^{\prime}\right)\right\rangle=\left\langle b^{\prime}, u\right\rangle
$$

with $u=l_{b}\left(u^{\prime}\right)$ a minimal projection in $b \mathcal{P}_{2,+} b$, so in $\mathcal{P}_{2,+}$.

Assuming Conjecture 5.11 and using Lemma 6.1, we get a non-trivial upper bound:

Conjecture 6.2. The minimal number $r$ of minimal projections generating the identity biprojection (i.e., $\left\langle u_{1}, \ldots, u_{r}\right\rangle=i d$ ) is less than the minimal length $\ell$ for an ordered chain of biprojections

$$
e_{1}=b_{0}<b_{1}<\cdots<b_{\ell}=i d
$$

such that $\left[b_{i}, b_{i+1}\right]$ is distributive (or better, top boolean).

Remark 6.3. We can deduce theorems from Conjecture 6.2, by adding some assumptions to $\left[b_{i}, b_{i+1}\right]$, according to Theorems 4.26 or 5.7 .

Remark 6.4. Let $(N \subset M)$ be any irreducible finite index subfactor. We can deduce a non-trivial upper bound for the minimal number of (algebraic) irreducible sub- $N$ - $N$-bimodules of $M$, generating $M$ as von Neumann algebra.

6.2. Back to the finite groups theory. As applications, we get a dual version of Theorem [2.5, and for any finite group $G$, we get a non-trivial upper bound for the minimal number of irreducible components for a faithful complex representation. The action of $G$ on the hyperfinite $\mathrm{II}_{1}$ factor $R$ is always assumed outer.

Theorem 6.5 (§226 [5]). A complex representation $V$ of a finite group $G$ is faithful if and only if any irreducible complex representation $W$ is equivalent to a subrepresentation of $V^{\otimes n}$, for some $n \geq 0$.

Definition 6.6. A group $G$ is linearly primitive if it admits a faithful irreducible complex representation. 
Definition 6.7. Let $W$ be a representation of a group $G, K$ a subgroup of $G$, and $X$ a subspace of $W$. Let the fixed-point subspace be

$$
W^{K}:=\{w \in W \mid k w=w, \forall k \in K\}
$$

and the pointwise stabilizer subgroup

$$
G_{(X)}:=\{g \in G \mid g x=x, \forall x \in X\}
$$

Definition 6.8. An interval $[H, G]$ is said to be linearly primitive if there is an irreducible complex representation $V$ of $G$ with $G_{\left(V^{H}\right)}=H$.

The group $G$ is linearly primitive iff the interval $[\{e\}, G]$ is so.

Lemma 6.9. Let $H$ be a core-free subgroup of $G$. Then $G$ is linearly primitive if $[H, G]$ is so.

Proof. Take $V$ as above. Now, $V^{H} \subset V$ so $G_{(V)} \subset G_{\left(V^{H}\right)}$, but $\operatorname{ker}\left(\pi_{V}\right)=G_{(V)}$, it follows that $\operatorname{ker}\left(\pi_{V}\right) \subset H$; but $H$ is a core-free subgroup of $G$, and $\operatorname{ker}\left(\pi_{V}\right)$ a normal subgroup of $G$, so $\operatorname{ker}\left(\pi_{V}\right)=\{e\}$, which means that $V$ is faithful on $G$, i.e. $G$ is linearly primitive.

Lemma 6.10. Let $p_{x} \in \mathcal{P}_{2,+}\left(R^{G} \subseteq R\right)$ be a minimal projection on the one-dimensional subspace $\mathbb{C} x$ and $H$ a subgroup of $G$. Then

$$
p_{x} \leq b_{H}:=|H|^{-1} \sum_{h \in H} \pi_{V}(h) \Leftrightarrow H \subset G_{x} .
$$

Proof. If $p_{x} \leq b_{H}$ then $b_{H} x=x$ and $\forall h \in H$ we have that

$$
\pi_{V}(h) x=\pi_{V}(h)\left[b_{H} x\right]=\left[\pi_{V}(h) \cdot b_{H}\right] x=b_{H} x=x
$$

which means that $h \in G_{x}$, and so $H \subset G_{x}$. Conversely, if $H \subset G_{x}$ (i.e. $\left.\forall h \in H, \pi_{V}(h) x=x\right)$ then $b_{H} x=x$, which means that $p_{x} \leq b_{H}$.

Theorem 6.11. Let $[H, G]$ be an interval of finite groups. Then

- $(R \rtimes H \subseteq R \rtimes G)$ is w-cyclic if and only if $[H, G]$ is $H$-cyclic.

- $\left(R^{G} \subseteq R^{H}\right)$ is w-cyclic if and only if $[H, G]$ is linearly primitive.

Proof. By Proposition 4.21, $(R \rtimes H \subseteq R \rtimes G)$ is w-cyclic if and only if

$$
\exists u \in \mathcal{P}_{2,+}(R \subseteq R \rtimes G) \simeq \bigoplus_{g \in G} \mathbb{C} e_{g} \simeq \mathbb{C}^{G}
$$

minimal projection such that $\langle b, u\rangle=i d$, with $b=e_{R \rtimes H}^{R \rtimes G}$ and $r_{b}^{-1}(b * u * b)$ is a minimal projection; if and only if $\exists g \in G$ such that $\langle H, g\rangle=G$, because $u$ is of the form $e_{g}$ and $\forall g^{\prime} \in H g H, H g^{\prime} H=H g H$.

By Proposition 4.18, $\left(R^{G} \subseteq R^{H}\right)$ is w-cyclic if and only if

$$
\exists u \in \mathcal{P}_{2,+}\left(R^{G} \subseteq R\right) \simeq \bigoplus_{V_{i} \text { irr. }} \operatorname{End}\left(V_{i}\right) \simeq \mathbb{C} G
$$


minimal projection such that $\langle u\rangle=e_{R^{H}}^{R}$; if and only if, by Lemma 6.10, $H=G_{x}$ with $u=p_{x}$ the projection on $\mathbb{C} x \subseteq V_{i}\left(\right.$ with $\left.Z\left(p_{x}\right)=p_{V_{i}}\right)$.

Note that $H \subset G_{\left(V_{i}^{H}\right)} \subset G_{x}$ so $H=G_{\left(V_{i}^{H}\right)}$.

Corollary 6.12. The subfactor $\left(R^{G} \subseteq R\right)$ (resp. $\left.(R \subseteq R \rtimes G)\right)$ is $w$-cyclic if and only if $G$ is linearly primitive (resp. cyclic).

Examples 6.13. The subfactors $\left(R^{S_{4}} \subset R^{S_{2}}\right)$, its dual and $\left(R^{S_{3}} \subset R\right)$, are w-cyclic, but $\left(R \subset R \rtimes S_{3}\right)$ and $\left(R^{S_{4}} \subset R^{\langle(1,2)(3,4)\rangle}\right)$ are not.

By Theorem 6.11, the group-theoretic reformulation of Conjecture 5.11 on $\left(R^{G} \subseteq R^{H}\right)$ is the following dual version of Theorem 2.5.

Conjecture 6.14. Let $[H, G]$ be a distributive interval of finite groups. Then $\exists V$ irreducible complex representation of $G$ such that $G_{\left(V^{H}\right)}=H$.

If moreover $H$ is core-free, then $G$ is linearly primitive (Lemma 6.91).

Problem 6.15. Is a finite group $G$ linearly primitive iff there is a core-free subgroup $H$ such that the interval $[H, G]$ is bottom boolean?

By Theorem 6.5, Conjecture 6.2 on $\mathcal{P}\left(R^{G} \subseteq R\right)$ reformulates as follows:

Conjecture 6.16. The minimal number of irreducible components for a faithful complex representation of a finite group $G$ is less than the minimal length $\ell$ for an ordered chain of subgroups

$$
\{e\}=H_{0}<H_{1}<\cdots<H_{\ell}=G
$$

such that $\left[H_{i}, H_{i+1}\right]$ is distributive (or better, bottom boolean).

This provides a bridge linking combinatorics and representations in the theory of finite groups.

Remark 6.17. We can upgrade Conjecture 6.16 by taking for $H_{0}$ any core-free subgroup of $H_{1}$, instead of just $\{e\}$; we can also deduce theorems, by adding some assumptions to $\left[H_{i}, H_{i+1}\right]$, according to the grouptheoretic reformulation of Theorems 4.27 or 5.7. Note that a normal biprojection in $\mathcal{P}\left(R^{G} \subseteq R^{H}\right)$ is given by a subgroup $K \in[H, G]$ with $H g K=K g H \forall g \in G$, see [25, Proposition 3.3].

Remark 6.18. We can also formulate results for finite quantum groups (i.e. finite dimensional Kac algebras), where the biprojections correspond to the left coideal $\star$-subalgebras, see [8, Theorem 4.4].

\section{ACKNowledgments}

This work is supported by the Institute of Mathematical Sciences, Chennai. The author is grateful to Vaughan Jones, Dietmar Bisch, 
Scott Morrison and David Evans for their recommendation for a postdoc at the IMSc. Thanks to my hosts V.S. Sunder and Vijay Kodiyalam, and to Zhengwei Liu, Feng Xu, Keshab Chandra Bakshi and Mamta Balodi, for useful advices and fruitful exchanges.

\section{REFERENCES}

[1] Narjess Afzaly, Scott Morrison, and David Penneys, The classification of subfactors with index at most $5 \frac{1}{4}$. arXiv:1611.05811.

[2] M. Asaeda and U. Haagerup, Exotic subfactors of finite depth with Jones indices $(5+\sqrt{13}) / 2$ and $(5+\sqrt{17}) / 2$, Comm. Math. Phys. 202 (1999), no. 1 , 1-63, DOI 10.1007/s002200050574. MR1686551 (2000c:46120)

[3] Keshab Bakshi, Intermediate planar algebra revisited, 31pp. arXiv:1611.05811.

[4] Dietmar Bisch, A note on intermediate subfactors, Pacific J. Math. 163 (1994), no. 2, 201-216, DOI 10.2140/pjm.1994.163.201. MR1262294 (95c:46105)

[5] William Burnside, Theory of groups of finite order. Second edition, Cambridge University Press, 1911.

[6] Masaki Izumi, The structure of sectors associated with Longo-Rehren inclusions. II. Examples, Rev. Math. Phys. 13 (2001), no. 5, 603-674, DOI 10.1142/S0129055X01000818. MR1832764 (2002k:46161)

[7] — Characterization of isomorphic group-subgroup subfactors, Int. Math. Res. Not. 34 (2002), 1791-1803, DOI 10.1155/S107379280220402X. MR1920326 (2003f:46100)

[8] Masaki Izumi, Roberto Longo, and Sorin Popa, A Galois correspondence for compact groups of automorphisms of von Neumann algebras with a generalization to Kac algebras, J. Funct. Anal. 155 (1998), no. 1, 25-63, DOI 10.1006/jfan.1997.3228. MR1622812 (2000c:46117)

[9] Vaughan F. R. Jones, Actions of finite groups on the hyperfinite type $\mathrm{II}_{1}$ factor, Mem. Amer. Math. Soc. 28 (1980), no. 237, v+70, DOI 10.1090/memo/0237. MR587749 (81m:46094)

[10] _ Index for subfactors, Invent. Math. 72 (1983), no. 1, 1-25, DOI 10.1007/BF01389127. MR696688 (84d:46097)

[11] Vaughan F. R. Jones and V. S. Sunder, Introduction to subfactors, London Mathematical Society Lecture Note Series, vol. 234, Cambridge University Press, Cambridge, 1997. MR1473221 (98h:46067)

[12] Vaughan F. R. Jones, Planar algebras, I, arXiv:math/9909027 (1999), 122pp. to appear in New Zealand Journal of Mathematics.

[13] Vaughan F. R. Jones, Scott Morrison, and Noah Snyder, The classification of subfactors of index at most 5, Bull. Amer. Math. Soc. (N.S.) 51 (2014), no. 2, 277-327, DOI 10.1090/S0273-0979-2013-01442-3. MR3166042

[14] Vijay Kodiyalam and V. S. Sunder, The subgroup-subfactor, Math. Scand. 86 (2000), no. 1, 45-74. MR1738515 (2001b:46103)

[15] _ On Jones' planar algebras, J. Knot Theory Ramifications 13 (2004), no. 2, 219-247, DOI 10.1142/S021821650400310X. MR2047470 (2005e:46119)

[16] Zeph A. Landau, Intermediate subfactors, 1998. Thesis (Ph.D.)-University of California at Berkeley. 
[17] _ Exchange relation planar algebras, Proceedings of the Conference on Geometric and Combinatorial Group Theory, Part II (Haifa, 2000), 2002, pp. 183-214, DOI 10.1023/A:1021296230310. MR1950890 (2003k:46091)

[18] Zhengwei Liu, Exchange relation planar algebras of small rank, Trans. Amer. Math. Soc. 368 (2016), no. 12, 8303-8348, DOI 10.1090/tran/6582. MR3551573

[19] Masahiro Nakamura and Zirô Takeda, On the fundamental theorem of the Galois theory for finite factors., Proc. Japan Acad. 36 (1960), 313-318. MR0123926 (23 \#A1247)

[20] Øystein Ore, Structures and group theory. II, Duke Math. J. 4 (1938), no. 2, 247-269, DOI 10.1215/S0012-7094-38-00419-3. MR1546048

[21] Sebastien Palcoux, Non- "weakly group theoretical" integral fusion categories?, MathOverflow. http://mathoverflow.net/q/132866 (version: 2016-07-26).

[22] Sebastien Palcoux, Ore's theorem for cyclic subfactor planar algebras and applications, arXiv:1505.06649v10, 50pp.

[23] Emily Peters, A planar algebra construction of the Haagerup subfactor, Internat. J. Math. 21 (2010), no. 8, 987-1045, DOI 10.1142/S0129167X10006380. MR2679382 (2011i:46077)

[24] Richard P. Stanley, Enumerative combinatorics. Volume 1, 2nd ed., Cambridge Studies in Advanced Mathematics, vol. 49, Cambridge University Press, Cambridge, 2012. MR2868112

[25] Tamotsu Teruya, normal intermediate subfactors, J. Math. Soc. Japan 50 (1998), no. 2, 469-490, DOI 10.2969/jmsj/05020469. MR1613172 (99e:46080)

[26] Yasuo Watatani, Lattices of intermediate subfactors, J. Funct. Anal. 140 (1996), no. 2, 312-334, DOI 10.1006/jfan.1996.0110. MR1409040 (98c:46134)

[27] Feng Xu, On a problem about tensor products of subfactors, Adv. Math. 246 (2013), 128-143, DOI 10.1016/j.aim.2013.06.026. MR3091803

Institute of Mathematical Sciences, Chennai, India

E-mail address: sebastienpalcoux@gmail.com 